ESJ Natural/Life/Medical Sciences

\title{
Etude Comparative des Toxicités Cellulaires et Aigües de Ageratum conyzoides L. et de Acanthospermum hispidum DC
}

\author{
Camara Djeneb,
}

Université Félix Houphouet Boigny, Ufr Biosciences, Laboratoire de

Botanique, Abidjan, Côte d'Ivoire

Université de Grenoble Rhone alpes, Laboratoire TimC Imag, Domaine de la

Merci, La Tronche cedex-France

Yapi Adon Basile,

Université Félix Houphouet Boigny, Ufr Biosciences, Laboratoire de

Botanique, Abidjan, Côte d'Ivoire

Fofié N'guessan Bra Yvette,

Université Félix Houphouet Boigny, UFR Sciences Pharmaceutiques Et Biologiques, Laboratoire De Pharmacognosie, Botanique Et Cryptogamie,

Côte d'Ivoire

\section{Ouattara Katinan Etienne,} Zirihi Guédé Noël,

Université Félix Houphouet Boigny, Ufr Biosciences, Laboratoire de Botanique, Abidjan, Côte d'Ivoire

Doi:10.19044/esj.2021.v17n40p74

Submitted: 30 July 2021

Accepted: 16 August 2021

Published: 30 November 2021
Copyright 2021 Author(s)

Under Creative Commons BY-NC-ND

4.0 OPEN ACCESS

Cite As:

Djeneb C., Basile Y.A., Yvette B.N.F., Etienne O.K \& Noël Z.G., (2021). Etude Comparative des Toxicités Cellulaires et Aigües de Ageratum conyzoides L. et de Acanthospermum hispidum DC. European Scientific Journal, ESJ, 17(40), 74.

https://doi.org/10.19044/esj.2021.v17n40p74

\section{Résumé}

Dans la recherche de nouveaux traitements thérapeutiques contre les maladies endémiques et récurrentes ; et dans le but de rassurer la population sur l'utilisation des médicaments à base de plantes, une étude toxicologique de deux adventices médicinales ivoiriennes : Ageratum conyzoides et Acanthospermum hispidum, a été faite. Cette étude consistait à la mise en évidence de la toxicité cellulaire sur les cellules humaines HFF et la toxicité aiguë sur des souris, des extraits éthanoliques $70 \%$ de ces deux plantes. L'extrait éthanolique $70 \%$ de A. hispidum n'est pas toxique, ni pour les 
cellules humaines aux concentrations de 125 à $1000 \mu \mathrm{g} / \mathrm{mL}$, ni pour les souris aux doses de 0-45 $000 \mathrm{mg} / \mathrm{kg} / \mathrm{Vo}$. Aucun signe de changement de comportement n'a été observé à ces mêmes doses. Par contre, l'extrait éthanolique $70 \%$ de $\mathrm{A}$. conyzoides est légèrement toxique sur les cellules HFF en prolifération à $1000 \mu \mathrm{g} / \mathrm{mL}$ (taux de viabilité de $40 \%$ ). Cet extrait ne présente pas de toxicité sur les souris à des doses inférieures à 15000 $\mathrm{mg} / \mathrm{kg} / \mathrm{Vo}$. Cependant aux doses supérieures à $15000 \mathrm{mg} / \mathrm{kg} / \mathrm{Vo}$ bien qu'aucune mortalité de souris n’a été observée, des troubles symptomatiques, des difficultés motrices, une dyspnée et une somnolence chez les souris traitées ont été constatés. Ainsi, ces deux adventices ne présentent pas de toxicité avérée, ce qui justifie leur utilisation dans la médecine traditionnelle.

Mots clés: Acanthospermum hispidum, Ageratum conyzoides, Cellules HFF, Toxicité

\section{Comparative Study of Cellular and Acute Toxicity of Ageratum conyzoides $\mathrm{L}$ and Acanthospermum hispidum CD}

\section{Camara Djeneb,}

Université Félix Houphouet Boigny, Ufr Biosciences, Laboratoire de Botanique, Abidjan, Côte d'Ivoire

Université de Grenoble Rhone alpes, Laboratoire TimC Imag, Domaine de la Merci, La Tronche cedex-France

\section{Yapi Adon Basile,}

Université Félix Houphouet Boigny, Ufr Biosciences, Laboratoire de

Botanique, Abidjan, Côte d'Ivoire

Fofié N'guessan Bra Yvette,

Université Félix Houphouet Boigny, UFR Sciences Pharmaceutiques Et Biologiques, Laboratoire De Pharmacognosie, Botanique Et Cryptogamie,

Côte d'Ivoire

\section{Ouattara Katinan Etienne,} Zirihi Guédé Noël,

Université Félix Houphouet Boigny, Ufr Biosciences, Laboratoire de

Botanique, Abidjan, Côte d'Ivoire

Abstract

Due to the search for new therapeutic treatments against endemic and recurrent diseases, and in order to reassure the population about the use of herbal medicines, a toxicological study of two Ivorian medicinal weeds, Ageratum conyzoides and Acanthospermum hispidum, was carried out. This study focuses on the demonstration of cellular toxicity on human HFF cells 
and acute toxicity on mice, i.e., ethanolic $70 \%$ extracts of the two plants. The ethanolic $70 \%$ extract of $A$. hispidum is not toxic, neither to human cells at concentrations of 125 to $1000 \mu \mathrm{g} / \mathrm{mL}$ nor to mice at doses of $0-45.000$ $\mathrm{mg} / \mathrm{kg} / \mathrm{vo}$. No signs of behavioral change were seen at these same doses. On the other hand, the ethanolic $70 \%$ extract of $A$. conyzoides is slightly toxic on proliferating HFF cells at $1000 \mu \mathrm{g} / \mathrm{mL}$ (viability rate of $40 \%$ ). This extract does not exhibit toxicity in mice at doses of less than $15,000 \mathrm{mg} / \mathrm{kg} / \mathrm{Vo}$. At doses above $15.000 \mathrm{mg} / \mathrm{kg} / \mathrm{Vo}$, although no mouse mortality was observed, symptomatic disturbances, motor difficulties, dyspnea, and drowsiness in the treated mice were observed. These two weeds do not exhibit proven toxicity, which justifies their use in traditional medicine.

Keywords: Acanthospermum hispidum, Ageratum conyzoides, HFF cells, toxicity

\section{Introduction}

Les difficultés liées au traitement de diverses pathologies, en occurrence, l'asthme, le diabète, la diarrhée, la fièvre typhoïde, l'hypertension artérielle, le paludisme, l'hémorroïde, et qui sont en train de dévaster notre monde, ont contraints nombreux scientifiques à rechercher des thérapeutiques nouvelles, moins onéreuses et faciles d'accès, dans l'arsenal des plantes du patrimoine commun (N'Guessan, 2008). Ainsi, dans le but de contribuer à la réduction de la mortalité liée à certaines affections et à leurs facteurs de risques, l'OMS encourage, dans son rapport AFR/RC57/7, les pays africains à soutenir et à financer la recherche en y intégrant la médecine traditionnelle (OMS, 2007). C'est dans ce souci qu'une enquête ethnobotanique menée, a permis de découvrir deux espèces de plantes qualifiées de mauvaises herbes (Ageratum conyzoides L. et Acanthospermum hispidum DC.), d'une grande efficacité, souvent sollicitées en médecine traditionnelle, par les tradithérapeutes du district d'Abidjan, dans le traitement de ces pathologies courantes et difficiles à éradiquer (Yapi et al., 2015). Si les effets thérapeutiques de ces plantes ont déjà fait l'objet de diverses études (Anani et al., 2000 ; Kamanzi et al., 2002 ; Fleischer et al., 2003 ; Hoffman et al., 2004 ; Gafon et al., 2012 ; Camara et al., 2019), aucune étude toxicologique à la fois sur les cellules humaines et les souris s'y rapportant n'a jamais été menée, à notre connaissance. Ekundayo et al. (1988), dans leurs travaux, se sont intéressés à l'étude cytotoxique de $A$. conyzoides uniquement. De même, Diarra (2006) s'est intéressé seulement à la toxicité aiguë de $A$. hispidum dans ses travaux. Or, l'étude de la toxicité d'une substance implique divers aspects. Selon Lapointe (2004), la cytotoxicité est la propriété d'un agent chimique ou biologique à être toxique pour les cellules, allant éventuellement jusqu'à leur destruction. La toxicité aiguë est celle qui provoque la mort d'un organisme 
vivant ou de très graves troubles physiologiques après un court délai suivant l'absorption unique ou répétée d'une dose assez importante d'un composé nocif. D’après Bismuth et al. (1987), les intoxications aiguës représentent la première cause d'hospitalisation dans des pays développés et la deuxième cause de mortalité des individus de moins de 30 ans dans les pays en voie de développement. Ainsi, l'Homme est constamment exposé à la toxicité des produits auxquels il est en contact, surtout les médicaments. Il est donc indispensable que les essais de toxicité accompagnent les essais d'activités biologiques au cours de la sélection de nouvelles molécules, (Bismuth et al., 1987). Pour parvenir à des médicaments traditionnels améliorés, il est donc nécessaire d'assurer une préparation médicamenteuse correcte et sécurisée avant leurs mises à la disposition des patients. Pour cette raison, il convient de vérifier la toxicité aiguë et la toxicité cellulaire de la matière première employée pour la mise au point des médicaments. C'est ainsi que ces deux Asteraceae (Ageratum conyzoides L. et Acanthospermum hispidum DC.) à usages thérapeutiques traditionnels multiples et à activités pharmacologiques diverses, ont été retenues pour des tests de toxicité aiguë et de cytotoxicité.

\section{1- Matériel et méthodes}

\subsection{Matériel}

\subsubsection{Matériel végétal}

Le matériel végétal est constitué des rameaux feuillés de Ageratum conyzoides L. et Acanthospermum hispidum DC. Ces rameaux feuillés ont été récoltés dans le District d'Abidjan pour la préparation des extraits végétaux.

\section{I.1.2 Matériel animal}

Les animaux utilisés dans cette étude étaient constitués de 60 souris blanches de souche suisses (mâles et femelles) pesant 18-20 g. Ces animaux ont été fournis par l'animalerie de l'UFR des Sciences Pharmaceutiques et Biologiques de l'Université Félix HOUPHOUËT-BOIGNY. Ces souris ont été utilisées pour les tests de la toxicité aiguë.

Une lignée de cellule humaine HFF (Human Foreskin Fibroblast) a été également utilisée. Elle a été fournie par le Laboratoire Adaptation et Pathogénie des Microorganismes (LAPM) de Grenoble en France. Ce sont des cellules humaines issues de prépuce de nouveaux-nés. Elles ont la particularité de former un tapis cellulaire après plusieurs jours de culture (96 heures), on dit alors qu'elles sont confluentes, elles arrêtent de se diviser par inhibition de contact. Lorsque ces cellules sont en culture depuis seulement 24 heures, elles sont dans un état de mitose (ou cellules en division). Ces cellules ont été utilisées pour apprécier la cytotoxicité. 


\section{I.2 Méthodes}

\section{I.2.1 Préparation de l'extrait}

Les rameaux feuillés des deux Asteraceae, Ageratum conyzoides L. et Acanthospermum hispidum DC., récoltés et débarrassés de tous débris, ont été rincés à l'eau et séchés séparément à l'abri du soleil. Après séchage, chaque échantillon a été réduit en poudre fine grâce à un broyeur électrique. Cent grammes (100 g) de poudre de chaque drogue ont été homogénéisés dans un 1 litre d'une solution éthanol-eau (70/30) dans un Blender (Mixer) de marque Life's Superb (LS-317) à la température ambiante. L’homogénat obtenu est filtré successivement sur un carré de tissu blanc, sur du coton hydrophile puis sur du papier Watman. Après trois cycles d'extraction (extraction par épuisement), le volume du filtrat final de chaque drogue a été mis à l'étuve réglée à $50{ }^{\circ} \mathrm{C}$ pour éliminer le solvant d'extraction (Basile et al., 2015). L'évaporat sec de chaque drogue est récupéré sous forme de poudre et constitue l'extrait hydro-éthanolique $70 \%$ de chaque plante.

\section{I.2.2. Réalisation des tests de cytotoxicité}

L’étude de la toxicité a été inspirée de la méthode de Mossman (1983). Les deux types de cellules HFF (confluentes et en division) utilisées, ont été cultivés à $37^{\circ} \mathrm{C}$, sous $5 \%$ de $\mathrm{CO}_{2}$ dans un milieu D10 (Dulbecco Minimum Essential Medium, Gibco) additionné de sérum de veau fœtal $10 \%$; glutamine $1 \%$; pénicilline $50 \mathrm{U} \cdot \mathrm{mL}^{-1}$ et streptomycine $50 \mu \mathrm{g} \cdot \mu \mathrm{L}^{-1}$.

Pour mesurer la cytotoxicité de l'extrait éthanolique, les cellules HFF ont été ensemencées dans des plaques de 96 puits (CellStar) à raison de 3000 à 5000 cellules par puits dans $100 \mu \mathrm{L}$ de milieu D10. Par la suite, elles ont été exposées pendant 24 heures à différentes concentration $(0-1000 \mu \mathrm{g} / \mathrm{mL})$ en extrait de plante solubilisé dans du tampon PBS. Cela a été fait en triplicate. La viabilité a été déterminée à l'aide du bromure de 3-(4,5-diméthylthiazol-2yl)-2,5-diphenyl tetrazolium (MTT). L'anneau de tétrazolium qu'il contient est réduit en formazan par la succinate déshydrogénase mitochondriale des cellules métaboliquement actives, qui précipite et donne une couleur violette. La quantité du précipité formé est proportionnelle au nombre de cellules vivantes. Dans chaque puits, le MTT est ajouté à une concentration de 500 $\mu \mathrm{g} / \mathrm{mL}$ et incubé pendant 3 heures à $37^{\circ} \mathrm{C}$. Les cristaux de formazan sont solubilisés dans du diméthylsulfoxyde (DMSO) $10 \mathrm{mM}$. La mesure de la densité optique à $544 \mathrm{~nm}$ a été faite à l'aide d'un spectrophotomètre Safir (Tecan). Cette mesure de l'absorbance a permis de déterminer la quantité relative de cellules vivantes et actives métaboliquement. Les résultats ont été exprimés en pourcentage de viabilité par rapport au contrôle sans extrait de plante à l'aide de la formule suivante :

Taux viabilité $=($ Abs544 nm extrait $/$ Abs544 nm témoin $) \times 100$ 


\section{I.2.3. Etude de la toxicité aigüe}

La détermination de cette toxicité s'est faite selon la méthode de Lichtfield et Wilcoxon (1949). Les 60 souris utilisées, ont été mises à jeûn pendant 12 heures avant les tests. Elles ont été acclimatées pendant une semaine avant le début de l'expérience. Elles ont été logées dans des cages en aluminium de taille moyenne et ont été réparties en 6 lots de 10 souris chacun dont un lot témoin. Les cages ont été placées dans une salle bien aérée. Cinq doses d'extrait éthanolique de chacune des deux plantes: Ageratum conyzoides et Acanthospermum hispidum, ont été testées (9000, 11250, 15000, 22500 et $45000 \mathrm{mg} / \mathrm{kg}$ de poids corporel). Ce qui correspondait à des concentrations respectives de 300, 375, 500, 750 et $1500 \mathrm{mg} / \mathrm{mL}$. Les souris de chaque lot-test ont reçu une concentration donnée par gavage gastrique à raison de $10 \mathrm{~mL} / \mathrm{kg}$ de poids corporel tandis que les souris du lot témoin n'ont reçu que de l'eau distillée à raison de $10 \mathrm{~mL} / \mathrm{kg}$ de poids corporel. Deux heures plus tard, les souris ont eu droit à une alimentation normale. Le taux de mortalité et tous les signes cliniques de toxicité au sein de chaque lot ont été notés. Les souris sont observées à 1 heure, 2 heures, 4 heures, 12 heures, 24 heures, 2 jours, 3 jours, 6 jours et 12 jours.

Certains paramètres sont évalués :

- la dose maximale tolérée (DMT);

- la dose létale $100 \%\left(\mathrm{DL}_{100}\right)$;

- la dose létale $50 \%$ (DL50).

Ce dernier paramètre tient compte de : la $\mathrm{DL}_{100}$; la moyenne de la somme des morts entre 2 doses successives ; la différence entre 2 doses successives et la moyenne du nombre d'animaux utilisés par lot. La formule de Karber et Berhens (1935) est utilisée pour le calcul de la $\mathrm{DL}_{50}$ :

$$
\mathrm{DL}_{50}=\mathrm{DL}_{100}-\Sigma(\mathrm{a} \mathrm{X} \mathrm{b}) / \mathrm{n}
$$

- $\mathrm{DL}_{100}=$ dose minimum toujours mortelle

- $\quad \sum=$ moyenne de la somme des morts entre 2 doses successives

- $\quad \mathrm{a}=$ différence entre 2 doses successives

- $\quad \mathrm{b}$ = la moyenne du nombre d'animaux utilisés par lot

- $\mathrm{n}$ = moyenne d'animaux utilisés

Le comportement des souris est observé à ces différents temps, les troubles symptomatiques suivant sont recherchés : difficultés motrices ; toilettage ; somnolence ; dyspnée ; mort.

\section{Résultats}

\section{II.1. Activité cytotoxique}

La Figure 1 montre l'effet de l'extrait éthanolique 70 \% de Ageratum conyzoides sur la viabilité des cellules humaines HFF. Pour les cellules confluentes, on observe au début de l'expérience une augmentation du pourcentage de survie des cellules de $100 \%$ à $128 \%$ à une concentration de 
$125 \mu \mathrm{g} / \mathrm{mL}$ avant de constater une baisse du pourcentage de survie des cellules à $97 \%$ à une concentration de $1000 \mu \mathrm{g} / \mathrm{mL}$. Pour les cellules en prolifération, on observe une baisse du pourcentage de viabilité de $60 \%$ à la concentration en extrait de plante de $1000 \mu \mathrm{g} / \mathrm{mL}$. Lorsque, les cellules sont en prolifération, A. conyzoides a un effet négatif sur la viabilité cellulaire. Par contre, pour les cellules en arrêt de mitose (confluentes), $A$. conyzoides à un effet positif sur la viabilité cellulaire. On en déduit que l'extrait éthanolique $70 \%$ de $A$. conyzoides n'est pas toxique sur les cellules humaines HFF en arrêt de mitose mais il est toxique pour les cellules humaines HFF en mitose aux concentrations testées.

Les résultats des tests de toxicité de l'extrait éthanolique $70 \%$ de Acanthospermum hispidum sur les cellules HFF, sont résumés à la Figure 2. Pour les cellules confluentes comme les cellules en prolifération, on observe au début de l'expérience, une baisse du taux de survie des cellules à $84 \%$ pour les cellules confluentes et à $62 \%$ pour les cellules en prolifération à une concentration de $125 \mu \mathrm{g} / \mathrm{mL}$ d'extrait, puis on constate une légère augmentation du pourcentage de survie des cellules à $93 \%$ pour les cellules confluente et à $81 \%$ pour les cellules en prolifération à une concentration de $1000 \mu \mathrm{g} / \mathrm{mL}$ d'extrait. On en déduit que l'extrait éthanolique $70 \%$ de $A$. hispidum montre peu d'effet toxique sur les cellules humaines aux concentrations étudiées.

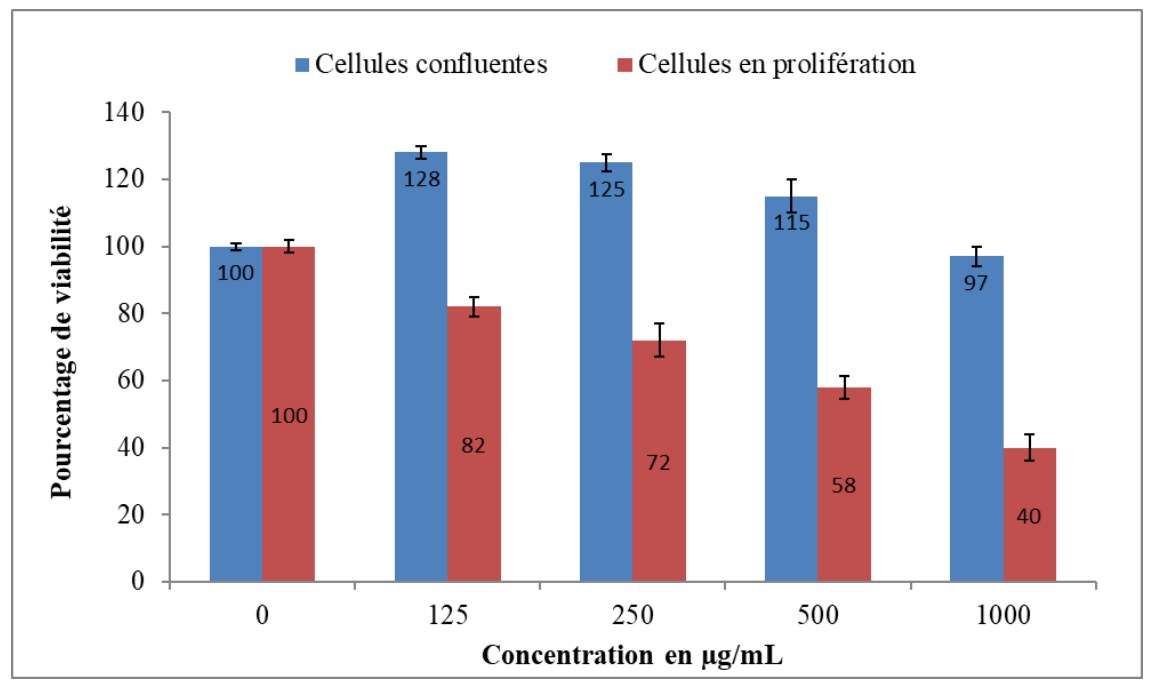

Figure 1. Effet de différentes concentrations en extrait éthanolique de A. conyzoides sur la viabilité des cellules humaines HFF. 


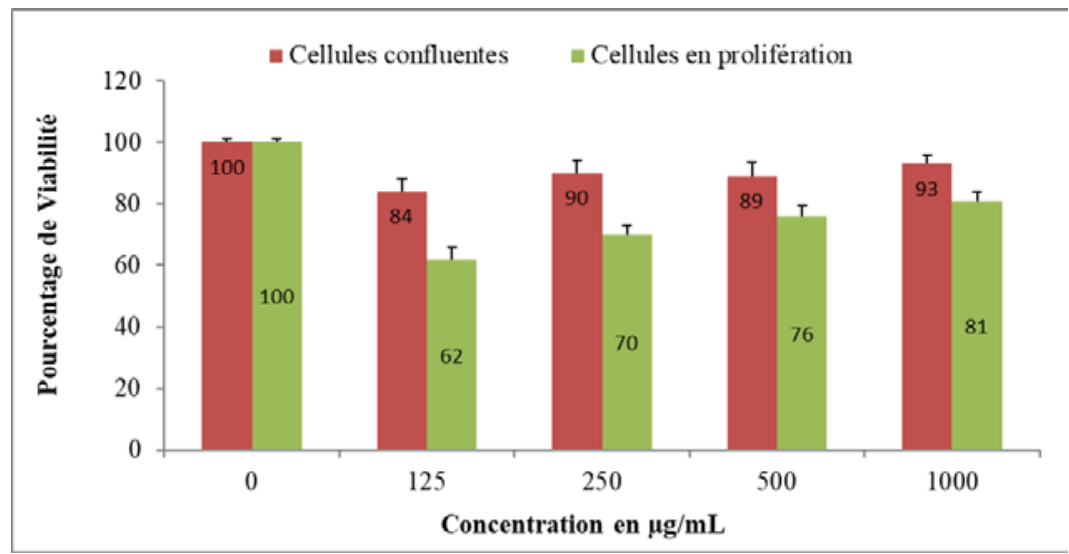

Figure 2. Effet de différentes concentrations en extrait éthanolique de A. hispidum sur la viabilité des cellules humaines HFF.

\section{II.2. Toxicité aigüe}

\section{II.2.1. Détermination de la DL50}

L'étude de la toxicité aiguë, après l'administration séparée par voie orale des extraits éthanoliques de Ageratum conyzoides et de Acanthospermum hispidum aux différents lots de souris, n’a enregistré aucune mort. La plus forte concentration d'extrait éthanolique administrée étant de $1500 \mathrm{mg} / \mathrm{mL}$ à raison de $10 \mathrm{~mL} / \mathrm{kg}$ de poids corporel, soit une dose maximale de $45000 \mathrm{mg} / \mathrm{kg}$ de poids corporel. L'absence de décès de souris traitées n'a pas permis de déterminer la $\mathrm{DL}_{50}$ (Tableau 1 et 2).

Tableau 1. Observation des caractéristiques toxicologiques après gavage séparé des souris avec l'extrait éthanolique de Ageratum conyzoides

\begin{tabular}{|c|c|c|c|c|c|c|}
\hline & Lot 1 & Lot 2 & Lot 3 & Lot 4 & Lot 5 & Lot 6 \\
\hline $\begin{array}{c}\text { Substance } \\
\text { administrée }\end{array}$ & $\begin{array}{c}\text { Eau } \\
\text { Distillée }\end{array}$ & Extrait & Extrait & Extrait & Extrait & Extrait \\
\hline $\begin{array}{c}\text { Concentration } \\
(\mathrm{mg} / \mathrm{mL})\end{array}$ & $0,6 \mathrm{~mL} / 20 \mathrm{~g}$ & 300 & 375 & 500 & 750 & 1500 \\
\hline $\begin{array}{c}\text { Dose } \\
\text { correspondante } \\
\text { (mg/Kg/Vo) }\end{array}$ & 00 & $\mathbf{9 0 0 0}$ & 11250 & 15000 & 22500 & $\mathbf{4 5 0 0 0}$ \\
\hline $\begin{array}{c}\text { Nombre de } \\
\text { souris par lot }\end{array}$ & 10 & 10 & 10 & 10 & 10 & 10 \\
\hline $\begin{array}{c}\text { Nombre de } \\
\text { souris mortes }\end{array}$ & $\mathbf{0 0}$ & $\mathbf{0 0}$ & $\mathbf{0 0}$ & $\mathbf{0 0}$ & $\mathbf{0 0}$ & $\mathbf{0 0}$ \\
\hline Mortalité (\%) & 00 & 00 & 00 & 00 & 00 & 00 \\
\hline
\end{tabular}

Tableau 2. Observation des caractéristiques toxicologiques après gavage séparé des souris avec l'extrait éthanolique de Acanthospermum hispidum.

\begin{tabular}{|c|r|r|r|r|r|r|}
\hline & Lot 1 & Lot 2 & Lot 3 & Lot 4 & Lot 5 & Lot 6 \\
\hline $\begin{array}{c}\text { Substance } \\
\text { administrée }\end{array}$ & $\begin{array}{c}\text { Eau } \\
\text { Distillée }\end{array}$ & Extrait & Extrait & Extrait & Extrait & Extrait \\
\hline
\end{tabular}




\begin{tabular}{|c|c|c|c|c|c|c|}
\hline $\begin{array}{c}\text { Concentration } \\
(\mathrm{mg} / \mathrm{mL})\end{array}$ & $0,6 \mathrm{~mL} / 20 \mathrm{~g}$ & 300 & 375 & 500 & 750 & 1500 \\
\hline $\begin{array}{c}\text { Dose } \\
\text { correspondante } \\
(\mathrm{mg} / \mathrm{Kg} / \mathrm{Vo})\end{array}$ & 00 & $\mathbf{9 0 0 0}$ & 11250 & 15000 & 22500 & $\mathbf{4 5 0 0 0}$ \\
\hline $\begin{array}{c}\text { Nombre de } \\
\text { souris par lot }\end{array}$ & 10 & 10 & 10 & 10 & 10 & 10 \\
\hline $\begin{array}{c}\text { Nombre de } \\
\text { souris mortes }\end{array}$ & $\mathbf{0 0}$ & $\mathbf{0 0}$ & $\mathbf{0 0}$ & $\mathbf{0 0}$ & $\mathbf{0 0}$ & $\mathbf{0 0}$ \\
\hline Mortalité $(\%)$ & 00 & 00 & 00 & 00 & 00 & 00 \\
\hline
\end{tabular}

\section{II.2.2. Evaluation des paramètres comportementaux}

\section{- De 0 à $15000 \mathrm{mg} / \mathrm{kg} / \mathrm{Vo}$}

Les doses administrées par voie orale jusqu'à 15000 mg/kg/Vo, n’ont provoqué chez les souris aucun changement de comportement. Aucun signe de toxicité telle qu'une diminution de la sensibilité à la douleur ou au bruit ou à la locomotion n’a été observé (Tableau 3 et 4).

Tableau 3. Effets des extraits éthanoliques de Ageratum conyzoides sur quelques paramètres physiologiques chez les souris au cours du temps. Doses $<15000 \mathrm{mg} / \mathrm{kg} / \mathrm{Vo}$

\begin{tabular}{|c|c|c|c|c|c|c|c|c|c|}
\hline \multicolumn{9}{|c|}{ Souris avec extrait entre 0 - $15000 \mathrm{mg} / \mathrm{kg} / \mathrm{Vo}$} & \\
\hline Période & 1h & $2 \mathrm{~h}$ & $4 \mathrm{~h}$ & $12 \mathrm{~h}$ & $24 \mathrm{~h}$ & $2 \mathrm{j}$ & $4 \mathrm{j}$ & $6 \mathrm{j}$ & $12 \mathrm{j}$ \\
\hline Motricité & $\mathrm{N}$ & $\mathrm{N}$ & $\mathrm{N}$ & $\mathrm{N}$ & $\mathrm{N}$ & $\mathrm{N}$ & $\mathrm{N}$ & $\mathrm{N}$ & $\mathrm{N}$ \\
\hline Toilettage & $\mathrm{N}$ & $\mathrm{N}$ & $\mathrm{N}$ & $\mathrm{N}$ & $\mathrm{N}$ & $\mathrm{N}$ & $\mathrm{N}$ & $\mathrm{N}$ & $\mathrm{N}$ \\
\hline Somnolence & $\mathrm{N}$ & $\mathrm{N}$ & $\mathrm{N}$ & $\mathrm{N}$ & $\mathrm{N}$ & $\mathrm{N}$ & $\mathrm{N}$ & $\mathrm{N}$ & $\mathrm{N}$ \\
\hline Dyspnée & $\mathrm{N}$ & $\mathrm{N}$ & $\mathrm{N}$ & $\mathrm{N}$ & $\mathrm{N}$ & $\mathrm{N}$ & $\mathrm{N}$ & $\mathrm{N}$ & $\mathrm{N}$ \\
\hline Mort & 00 & 00 & 00 & 00 & 00 & 00 & 00 & 00 & 00 \\
\hline
\end{tabular}

$\mathbf{N}=$ Normale

Tableau 4. Effets des extraits éthanoliques de Acanthospermum hispidum sur quelques paramètres physiologiques chez les souris au cours du temps. Doses $<15000 \mathrm{mg} / \mathrm{kg} / \mathrm{Vo}$

\begin{tabular}{|c|c|c|c|c|c|c|c|c|c|}
\hline \multicolumn{10}{|c|}{ Souris avec extrait entre 0 - $15000 \mathrm{mg} / \mathrm{kg} / \mathrm{Vo}$} \\
\hline Période & $1 \mathrm{~h}$ & $2 \mathrm{~h}$ & $4 \mathrm{~h}$ & $12 \mathrm{~h}$ & $24 \mathrm{~h}$ & $2 \mathrm{j}$ & $4 \mathrm{j}$ & $6 j$ & $12 \mathrm{j}$ \\
\hline Motricité & $\mathrm{N}$ & $\mathrm{N}$ & $\mathrm{N}$ & $\mathrm{N}$ & $\mathrm{N}$ & $\mathrm{N}$ & $\mathrm{N}$ & $\mathrm{N}$ & $\mathrm{N}$ \\
\hline Toilettage & $\mathrm{N}$ & $\mathrm{N}$ & $\mathrm{N}$ & $\mathrm{N}$ & $\mathrm{N}$ & $\mathrm{N}$ & $\mathrm{N}$ & $\mathrm{N}$ & $\mathrm{N}$ \\
\hline Somnolence & $\mathrm{N}$ & $\mathrm{N}$ & $\mathrm{N}$ & $\mathrm{N}$ & $\mathrm{N}$ & $\mathrm{N}$ & $\mathrm{N}$ & $\mathrm{N}$ & $\mathrm{N}$ \\
\hline Dyspnée & $\mathrm{N}$ & $\mathrm{N}$ & $\mathrm{N}$ & $\mathrm{N}$ & $\mathrm{N}$ & $\mathrm{N}$ & $\mathrm{N}$ & $\mathrm{N}$ & $\mathrm{N}$ \\
\hline Mort & 00 & 00 & 00 & 00 & 00 & 00 & 00 & 00 & 00 \\
\hline
\end{tabular}

$\mathbf{N}=$ Normale

\section{- De $15000 \mathrm{mg} / \mathrm{kg} / \mathrm{Vo}$ à $45000 \mathrm{mg} / \mathrm{kg} / \mathrm{Vo}$}

Aux différentes doses de cet intervalle, l'administration par voie orale de l'extrait éthanolique $70 \%$ de Ageratum conyzoides a provoqué des troubles symptomatiques: des difficultés motrices, une dyspnée et une somnolence accrue chez les souris traitées. Au bout de 12 heures ces troubles symptomatiques disparaissent (Tableau 5). 
Cependant, pour l'extrait éthanolique 70 \% de Acanthospermum hispidum, aux différentes doses de cet intervalle, l'administration par voie orale de l'extrait n'a provoqué aucun trouble symptomatique (Tableau 6).

Tableau 5. Effets de l'extrait éthanolique de Ageratum conyzoides sur quelques paramètres physiologiques chez les souris au cours du temps. Doses $>15000 \mathrm{mg} / \mathrm{kg} / \mathrm{Vo}$

\begin{tabular}{|c|c|c|c|c|c|c|c|c|c|}
\hline \multicolumn{10}{|c|}{ Souris avec extrait entre $15000 \mathrm{mg} / \mathrm{kg} / \mathrm{Vo}$ et $45000 \mathrm{mg} / \mathrm{Kg} / \mathrm{Vo}$} \\
\hline Période & 1h & $2 \mathrm{~h}$ & $4 \mathrm{~h}$ & $12 \mathrm{~h}$ & $24 \mathrm{~h}$ & $2 \mathrm{j}$ & $4 \mathrm{j}$ & $6 \mathrm{j}$ & $12 \mathrm{j}$ \\
\hline motricité & $\mathrm{R}$ & $\mathrm{R}$ & $\mathrm{R}$ & $\mathrm{N}$ & $\mathrm{N}$ & $\mathrm{N}$ & $\mathrm{N}$ & $\mathrm{N}$ & $\mathrm{N}$ \\
\hline Toilettage & $\mathrm{D}$ & $\mathrm{D}$ & $\mathrm{D}$ & $\mathrm{N}$ & $\mathrm{N}$ & $\mathrm{N}$ & $\mathrm{N}$ & $\mathrm{N}$ & $\mathrm{N}$ \\
\hline Somnolence & $\mathrm{Au}$ & $\mathrm{Au}$ & $\mathrm{Au}$ & $\mathrm{N}$ & $\mathrm{N}$ & $\mathrm{N}$ & $\mathrm{N}$ & $\mathrm{N}$ & $\mathrm{N}$ \\
\hline Dyspnée & $\mathrm{Au}$ & $\mathrm{Au}$ & $\mathrm{Au}$ & $\mathrm{N}$ & $\mathrm{N}$ & $\mathrm{N}$ & $\mathrm{N}$ & $\mathrm{N}$ & $\mathrm{N}$ \\
\hline Mort & 00 & 00 & 00 & 00 & 00 & 00 & 00 & 00 & 00 \\
\hline
\end{tabular}

$\mathbf{N}=$ Normale ; $\mathbf{D}=$ diminué ; $\mathbf{R}=$ réduit ; $\mathbf{A u}=$ Augmenter

Tableau 6. Effets de l'extrait de $A$. hispidum sur quelques paramètres physiologiques chez les souris au cours du temps. Doses $>15000 \mathrm{mg} / \mathrm{kg} / \mathrm{Vo}$

\begin{tabular}{|c|c|c|c|c|c|c|c|c|c|}
\hline \multicolumn{10}{|c|}{ Souris avec extrait entre $15000 \mathbf{~ m g} / \mathbf{k g} /$ Vo et $45000 \mathbf{~ m g} / \mathbf{K g} / \mathbf{V o}$} \\
\hline Période & $1 \mathrm{~h}$ & $2 \mathrm{~h}$ & $4 \mathrm{~h}$ & $12 \mathrm{~h}$ & $24 \mathrm{~h}$ & $2 \mathrm{j}$ & $4 \mathrm{j}$ & $6 \mathrm{j}$ & $12 \mathrm{j}$ \\
\hline motricité & $\mathrm{N}$ & $\mathrm{N}$ & $\mathrm{N}$ & $\mathrm{N}$ & $\mathrm{N}$ & $\mathrm{N}$ & $\mathrm{N}$ & $\mathrm{N}$ & $\mathrm{N}$ \\
\hline Toilettage & $\mathrm{N}$ & $\mathrm{N}$ & $\mathrm{N}$ & $\mathrm{N}$ & $\mathrm{N}$ & $\mathrm{N}$ & $\mathrm{N}$ & $\mathrm{N}$ & $\mathrm{N}$ \\
\hline Somnolence & $\mathrm{N}$ & $\mathrm{N}$ & $\mathrm{N}$ & $\mathrm{N}$ & $\mathrm{N}$ & $\mathrm{N}$ & $\mathrm{N}$ & $\mathrm{N}$ & $\mathrm{N}$ \\
\hline Dyspnée & $\mathrm{N}$ & $\mathrm{N}$ & $\mathrm{N}$ & $\mathrm{N}$ & $\mathrm{N}$ & $\mathrm{N}$ & $\mathrm{N}$ & $\mathrm{N}$ & $\mathrm{N}$ \\
\hline Mort & 00 & 00 & 00 & 00 & 00 & 00 & 00 & 00 & 00 \\
\hline
\end{tabular}

$\mathbf{N}=$ Normale

\section{Discussion}

Ageratum conyzoides L. et Acanthospermum hispidum DC., deux plantes de la famille botanique des Asteraceae et qualifiées de plantes envahissantes, sont très sollicitées dans le traitement de nombreuses maladies récurrentes.

La présente étude a révélé que l'extrait éthanolique $70 \%$ de $A$. hispidum ne présente pas de nocivité avérée sur les cellules humaines HFF étudiées à $1000 \mu \mathrm{g} / \mathrm{mL}$. Par contre, l'extrait éthanolique $70 \%$ de $A$. conyzoides présente des effets légèrement toxiques sur les cellules humaines HFF en prolifération mais augmente l'activité de celles qui ne se divisent plus. Le test MTT permet de visualiser l'activité d'une enzyme mitochondriale: la succinate déshydrogénase (SDH). La SDH est une enzyme qui intervient dans la respiration mitochondriale, lorsque dans une cellule, la SDH est très active, cela implique que la cellule est métaboliquement très active (Béné, 2017). A. hispidum a très peu d'effet sur la viabilité cellulaire, puisque le pourcentage de viabilité a faiblement varié. Ce qui révèle l'innocuité de l'extrait éthanolique $70 \%$ de $A$. hispidum sur les cellules HFF. En effet, selon les travaux de Coularie (2012), lorsque la viabilité cellulaire est supérieure à 30 $\%$, la substance testée n'est pas toxique. Toutefois, cette viabilité cellulaire 
diminue davantage avec l'augmentation de la concentration de l'extrait. L'extrait éthanolique $70 \%$ de $A$. conyzoides quant à lui, provoque une augmentation du taux de viabilité pour les cellules confluentes. Dans ce cas précis, on suppose alors que la SDH des cellules confluentes est activée d'où une activité métabolique augmentée, qui se traduit par la hausse de la viabilité cellulaire. En outre, la forte baisse du taux de viabilité des cellules en prolifération observée avec cet extrait végétal, suppose la présence dans l'extrait de molécules qui bloquent la mitose. Toutefois, $A$. conyzoides ne présente pas de nocivité notoire puisqu'avec les deux types de cellules, on n'observe pas une variation de la viabilité cellulaire en dessous de $30 \%$ (Coularie, 2012). Nos résultats sont conformes à ceux de Ekundayo et al. (1988). Selon ces auteurs, A. conyzoides a un effet toxique sur les cellules en division alors qu'elle n'agit pas sur les cellules en fin de mitose.

D'après les résultats de l'étude de toxicité aiguë, on peut dire qu'avec les concentrations de l'extrait éthanolique de A. hispidum utilisées, aucune toxicité n’a été observée car aucune mort n'a été enregistrée au niveau des souris, même à la dose la plus élevée. La DL50 n'a pas pu être déterminée, car la dose la plus élevée n’a entraîné aucun décès. Cependant avec l'extrait éthanolique de $A$. conyzoides, aux doses supérieures à $15000 \mathrm{mg} / \mathrm{kg} / \mathrm{vo}$, des troubles symptomatiques, des difficultés motrices, une dyspnée et une somnolence ont été observé chez les souris traitées ; ces résultats sont en adéquation avec la toxicité cellulaire de $A$. conyzoides. Diarra (2006) avait montré que l'extrait aqueux de $A$. hispidum pris par voie orale n'est pas toxique pour les souris.

\section{Conclusion}

L'étude a porté sur l'évaluation de la toxicité cellulaire et de la toxicité aiguë des extraits éthanoliques de deux Asteraceae : Ageratum conyzoides L. et Acanthospermum hispidum DC. L'extrait éthanolique de A. hispidum n'est ni toxique pour les cellules HFF, ni pour les souris. Aucun signe de toxicité du point de vue comportemental n'a été observé. Cependant, l'extrait éthanolique de $A$. conyzoides présente un effet légèrement toxique sur les cellules humaines HFF lorsque celles-ci sont en prolifération. Certes, les doses des extraits supérieures à $15000 \mathrm{mg} / \mathrm{kg} /$ vo n'ont causé aucune mortalité des souris, mais ces doses ont provoqué des troubles symptomatiques, des difficultés motrices, une dyspnée et une somnolence chez les souris traitées avec l'extrait éthanolique de $A$. conyzoides. Cette étude pourrait être mise à profit pour le développement de médicaments traditionnels améliorés efficaces pouvant traiter certaines maladies récurrentes telles que le paludisme, les kystes ovariens, les pertes blanches, les maladies de la peau dans la limite des doses sures. 


\section{Remerciements}

Nous remercions le Programme d'Appui Stratégique à la Recherche Scientifique (PASRES) de la Côte d'Ivoire et l'Académie des Sciences, des Arts, des Cultures d'Afrique et des Diasporas Africaines pour les financements accordés pour la réalisation des travaux de recherche qui ont aboutis aux résultats publiés.

\section{Références}

1. Anani, K., Hudson, J. B., De Souza, C., Akpagana, K., Tower, G. H. N., Amason, J. T., \& Gbeassor, M. (2000). Investigation of medicinal plants of Togo for antiviral and antimicrobial activities. Pharmaceutical. Biology, 38 (1) : 40-45.

2. Basile, A. Y., Goueh, G., Djeneb, C., \& Guédé, N. Z. (2015). Etude botanique, evaluation de l'activite antifongique des feuilles de Acanthospermum hispidum DC., (Asteraceae) sur la croissance in vitro de Candida albicans et etude de la toxicite sur les cellules humaines HFF. European Scientific Journal, 11 (30) : 225 - 237.

3. Béné, K. (2017). Plantes médicinales du Gontougo (District du Zanzan, Côte d’Ivoire) : inventaire, évaluation des activités pharmacologiques de deux plantes et formulation d'une pommade dermatologique à partir de l'extrait hydroalcoolique de Bersama abyssinica fresen. (Melianthaceae). Thèse Unique de Doctorat, UFR Biosciences, Université Félix HOUPHOUËT-BOIGNY, Abidjan, Côte d'Ivoire, 198p.

4. Bismuth, C., Band, F., Conse, F., Fréjaville, P. P., \& Garnier, R. (1987). Toxicologie clinique. Flammarion Medicine Science, Paris, $956 \mathrm{p}$.

5. Camara, D., Coulibaly, K., Yapi, A. B., Bene, K., \& Zirihi, G. N. (2019). Botanical study, antiparasitic activity on Toxoplasma gondii and cytotoxicity of Ageratum conyzoides and Synedrella nodiflora, two medicinal Asteraceae. European Journal of Pharmaceutical and Medical Research, 6 (6): 103-109.

6. Coularie, M. P. (2012). Etude phytochimique et pharmacologique de plantes de Nouvelle-Calédonie à potentialités anti-dengue. Thèse en chimie des substances naturelles, Université de la Nouvelle-Calédoine, Nouméa, 290p.

7. Diarra, Y. (2006). Etude de la phytochimie et des activités biologiques de Acanthospermum hispidum DC. (Asteraceae) et Curculigo pilosa Schum. et Thonn. (Hypoxidaceae), deux plantes utilisées dans le traitement traditionnel de l'hypertrophie benigne de la prostate (HBP). Thèse de doctorat d'Etat, Université de Bamako, URF FMPOS, 143 p. 
8. Ekundayo, O., Laakso, I., \& Hiltunen, R. (1988). Essential oil of Ageratum conyzoides. Planta Medica, 54 (1): 55-57.

9. Fleischer, T. C., Ameade, E. P., \& Sawer, I. K. (2003). Antimicrobial activity of the leaves and flowering tops of Acanthospermum hispidum. Fitoterapia, 74 : 130-132.

10. Ganfon, H., Bero, J., Alembert, T., Tchinda, A. T., Fernand, G., Gbenou, J., Moudachirou, M., Michel, F. M., \& Quetin-Leclercq, J. (2012). Antiparasitic activities of two sesquiterpenic lactones isolated from Acanthospermum hispidum DC. Journal of Ethnopharmacology, $141:$ 411-417.

11. Hoffman, B. R., Delasalas, H., Blanco, K., Wiederhold, N., Lewis, R. E., \& Williams, L. (2004). Screening of antibacterial and antifungal activities of ten medicinal plants from Ghana. Pharmaceutical Biology, 42 (1): 13-17.

12. Kamanzi, A. K., Koné, M., Terreaux, C., Traoré, D., Hostettmann, K., \& Dosso, M. (2002). Evaluation of the antimicrobial potential of medicinal plants from the Ivory Coast. Phytotherapy. Research, 16: 497-502.

13. Karber, C. \& Brehrens, B. (1935). Wiesind Reihenversuche fur biologische Auswertungen am Zweckmässigst en Anzuordnen? Archiv fur Experimentelle Pathologie und Pharmakologie, 177 : 379-388.

14. Lapointe, G. (2004). Notions de toxicologie. Commission de la santé et de la sécurité du travail du Québec (CSST), $2^{\text {ème }}$ édition revue et augmentée, 69 p.

15. Lichtfield, J. F. \& Wilcoxon, F. A. (1949). A simplified method of evaluation doses effects experiments. Journal of Pharmacology and Experimental Therapeutics, 95 : 99-113.

16. Mossman, T. (1983). Rapid colorimetric assay for cellular growth and survival: application to prolif-eration and cytotoxicity assay. Journal of immunological Methods, 65 : 55-63

17. N'Guessan, K. (2008). Plantes médicinales et pratiques médicales traditionnelles chez les peuples Abbey et Krobou du Département d'Agboville (Côte d'Ivoire), Thèse de Doctorat d'Etat ès Sciences Naturelles, Spécialité Ethnobotanique, Université de Cocody-Abidjan (Côte-d'Ivoire), UFR Biosciences, Laboratoire de Botanique, $\mathrm{N}^{\circ}$ d'ordre : 561/2008, 335 p.

18. O.M.S. (2007). Prévention et contrôle du diabète : une stratégie pour la région africaine de l'OMS. Comité régional de l'Afrique. Cinquante-septième session Brazzaville, République du Congo, 27-31 Août 2007, 8p.

19. Yapi, A. B., Kassi, N. J., Fofié, N. B. Y., \& Zirihi, G. N. (2015). Etude ethnobotanique des Asteraceae médicinales vendues sur les marches 
du district autonome d'Abidjan (Côte d'Ivoire). International Journal of Biological and Chemical Sciences, 9(6): 2633-2647. 